\title{
Cytogenomic Delineation and Clinical Characterization of Three Cases of MECP2 Duplication Syndrome
}

Cristelle Chow ${ }^{1}$, Angeline H.M. Lai ${ }^{2,3}$, Maggie S. Brett ${ }^{4}$, Simon Ling ${ }^{1}$, Jung Sook Ha ${ }^{5}$, Eileen C.P. Lim ${ }^{4}$, Ee-Shien Tan ${ }^{2}$, and Ene-Choo Tan ${ }^{3,4^{\star}}$

${ }^{1}$ Neurology Service, KK Women's \& Children's Hospital, Singapore

${ }^{2}$ Genetics Service, KK Women's \& Children's Hospital, Singapore

${ }^{3}$ Paediatrics Academic Clinical Programme, SingHealth Duke-NUS Graduate Medical School, Singapore

${ }^{4} K K$ Research Centre, KK Women's \& Children's Hospital, Singapore

${ }^{5}$ Department of Laboratory Medicine, Keimyung University School of Medicine, Daegu, Korea

\begin{abstract}
The methyl-CpG-binding protein 2 gene (MECP2) on the $\mathrm{X}$ chromosome encodes an essential epigenetic regulator in human postnatal brain development. Increased dosage of MECP2 causes a severe syndromic form of intellectual disability, the MECP2 duplication syndrome. Males with this syndrome have a progressive neurological disorder, severe to profound intellectual disability, epilepsy and recurrent respiratory infections. We report three cases with copy number gain in Xq28 involving the MECP2 gene. The gains were detected by chromosomal microarray analysis and ranged in size from $300 \mathrm{~kb}$ to $4.96 \mathrm{Mb}$. The three boys were aged between 3 and 16 years old. All three had development delay and no speech. In addition, one patient was diagnosed with Lennox-Gastaut syndrome and another had a Dandy Walker variant. Their clinical features were compared with other reported cases. We concluded that all three patients' clinical features were due to the Xq28 duplication, which confirmed the utility of chromosomal microarray analysis as a first-tier test in patients with unexplained intellectual disability. With a specific genetic diagnosis, we were able to provide appropriate anticipatory guidance for these patients and their families.
\end{abstract}

Keywords: Chromosomal microarray analysis; Copy number gain; Duplication syndrome; MECP2; Xq28

\section{Introduction}

Mutations in $M E C P 2$ on Xq28 chromosome were identified as the cause of Rett syndrome in 1999 [1]. Subsequently, a submicroscopic duplication encompassing MECP2 was reported in 2005 in a boy with hypotonia, intellectual disability, absent speech, loss of purposeful hand movement and development of stereotypic hand movements [2]. The use of multiplex ligation-dependent probe amplification (MLPA), and more recently, chromosomal microarray analysis (CMA), has led to the identification of an increasing number of affected males and the recognition of the MECP2 duplication syndrome phenotype.

To date, more than 150 affected males have been described in published reports [3]. The reported clinical features in all cases include infantile hypotonia with progressive spasticity predominantly affecting the lower limbs, developmental delay leading to severe to profound intellectual disability, poor speech development, recurrent respiratory infections, and epilepsy [4]. Dysmorphic features which may be subtle include brachycephaly, large ears, midface retrusion and depressed nasal bridge. Autistic behaviors and problems with gastrointestinal motility have also been reported [5]. Studies suggest that MECP2 overexpression could have a deleterious effect on brain development and function in mouse models, manifesting as seizures, spasticity, hypoactivity and early death [6]. In this report, we describe the clinical features and genomic rearrangements in three patients with $M E C P 2$ duplication.

\section{Material and Methods}

\section{Patients' samples}

The studies were approved by the Singhealth Centralized Institutional Review Board which oversees all research conducted at KK Women's \& Children's Hospital. All three patients were evaluated at Genetics Service, KK Women's and Children's Hospital, Singapore. Peripheral blood was collected in EDTA tubes after written informed consent for CMA testing was obtained from the parents.

\section{CMA}

Genomic DNA was extracted from fresh peripheral blood using the Puregene DNA Isolation Kit (Qiagen GmbH, Hilden, Germany). Patients 1 and 2 were tested with the $400 \mathrm{~K}$ oligonucleotide Human CGH array while Patient 3 was tested with the $244 \mathrm{~K}$ oligonucleotide array (Agilent Technologies Inc., Santa Clara, USA). Reference DNA used was male human genomic DNA (Promega Corp., Madison, WI, USA). The arrays were processed according to the manufacturer's instructions and scanned with Agilent G2505C Microarray scanner at 5 micron resolution. Data was extracted from the scanned image using Agilent Feature Extraction (Version 10.7.31) and analyzed for copy number change using Agilent Genomic Workbench Lite (Edition 6.0.130.24). Genomic coordinates are based on genome build 36/hg18.

\section{Quantitative real-time PCR (qRT-PCR) analysis}

A pair of primers for $M E C P 2$ was used for confirmation of copy number gain and analysis of parental samples. Quantitative real-time PCR was performed using FastStart Universal SYBR Green Master

*Corresponding author: Ene-Choo Tan, KK Research Centre, KK Women's \& Children's Hospital, 100 Bukit Timah Road, Singapore 229899, Fax: 656394 3792; E-mail: tanec@bigfoot.com

Received July 23, 2015; Accepted August 26, 2015; Published September 04 2015

Citation: Chow C, Lai AHM, Brett MS, Ling S, Ha JS, et al. (2015) Cytogenomic Delineation and Clinical Characterization of Three Cases of MECP2 Duplication Syndrome. J Clin Med Genom 3: 125. doi: 10.4172/2472-128X.1000125

Copyright: $\odot 2015$ Chow C, et al. This is an open-access article distributed under the terms of the Creative Commons Attribution License, which permits unrestricted use, distribution, and reproduction in any medium, provided the original author and source are credited. 
(ROX) (Roche Diagnostics Deutschland GmbH, Mannheim, Germany) and the StepOnePlus real time PCR system (Applied Biosystems Incorporated, Foster City, CA, USA). Results were analyzed using Applied Biosystems StepOne software (version 2.1), and the data were normalized against the reference gene ZNF80.

\section{Results}

\section{Comparison of clinical features}

Clinical and demographic features and comparison with reported cases are summarized in Table 1.

\section{Case presentations}

\section{Patient 1}

Patient 1 is the offspring of non-consanguineous healthy Malay parents. He was delivered at term following an uneventful pregnancy via elective caesarean section with a birthweight of $2750 \mathrm{~g}$. He was admitted at 6 days of life for poor feeding and was found to have hypertrichosis and micrognathia. He had recurrent admissions from 14 to 19 months of age for viral bronchiolitis and had one episode of severe pneumonia requiring ventilator support at 18 months of age during which he developed a febrile generalized tonic-clonic seizure. He also had chronic constipation, failure to thrive, and gastroesophageal reflux. His development was globally delayed and he had generalized hypotonia. His karyotype was normal $(46, \mathrm{XY})$.

Ultrasound scans of the kidneys and spine showed bilateral pelviectasis and intraspinal lipomatosis in the sacral region, both of which were conservatively managed. Magnetic resonance imaging study of his brain demonstrated a thin corpus callosum and a Dandy Walker variant with an inferiorly deficient cerebellum, communication between the fourth ventricle and cisterna magna, prominent occipital and temporal horns of the lateral ventricle and a dilated third ventricle. He developed upper airway obstruction due to tracheal compression and required non-invasive ventilation during sleep from 2 years of age. His serum immunoglobulin levels were within normal limits and his chest $\mathrm{x}$-ray showed mediastinal lymphadenopathy of unknown cause. Presently 3 years old, he is able to roll over and sit with minimal support. Speech development is absent and he is fed via gastrostomy. His seizures are well controlled but he continues to have frequent respiratory infections.

\section{Patient 2}

Patient 2 was delivered at full term with a birthweight of $2630 \mathrm{~g}$ following an uneventful pregnancy. His mother is of mixed European and Asian ancestry, while his father is Caucasian. He initially presented at 16 months of age with global developmental delay. Clinical examination showed plagiocephaly, generalized hypotonia, and normal deep tendon reflexes. There were no specific dysmorphic features. He sat without support at 2 years of age, but was never able to walk independently or speak more than 3 single words. As he grew older, he was noted to have increased lower limb spasticity and started to develop tight tendoarchilles and ankle contractures. He was frequently admitted for lower respiratory tract infections. He was admitted with tonic seizures and drop attacks at 9 years old. He was also noted to have repetitive purposeless movements such as hand wringing and body rocking. His electroencephalogram (EEG) showed a slow background with multifocal epileptiform discharges and he was started on carbamazepine, which has resulted in satisfactory seizure control. Currently at 12 years old, he has no speech, is wheelchair-bound but is fed orally, and he continues to have recurrent respiratory infections.

\section{Patient 3}

Patient 3 was delivered at term to healthy non-consanguineous Chinese parents following an uncomplicated pregnancy with a birthweight of $2700 \mathrm{~g}$. He had a simple febrile seizure at 18 months old. When he was initially evaluated at 9 years old, he was not able to speak or feed himself, and walked with a slight shuffling gait. His mother reported frequent episodes of drop attacks described as a sudden loss of tone while standing or sitting, with rapid spontaneous recovery. He had large ears and thick vermillion of upper and lower lips. His tone and reflexes were normal and he had non-purposeful repetitive movements of the upper limbs.

His EEG at 11 years old demonstrated diffuse slow spike waves as well as generalized and multifocal spike wave and poly-spike wave complexes. Based on his clinical features and EEG findings, he was diagnosed with Lennox-Gastaut syndrome. Magnetic resonance imaging (MRI) study of his brain performed at 12 years old was normal. He gradually developed medically refractory epilepsy and underwent a corpus callosotomy at 13 years of age to improve seizure control Although his seizure control improved, his post-operative period was complicated by a right subdural haemorrhage and bilateral basal ganglia infarcts, resulting in significant deconditioning. His neurorehabilitation

\begin{tabular}{|c|c|c|c|c|}
\hline Clinical characteristics & Patient 1 & Patient 2 & Patient 3 & Frequency or ranges in reported cases \\
\hline Gender & Male & Male & Male & N.A. \\
\hline Age when last reviewed & 3 years old & 12 years old & 16 years old & N.A. \\
\hline Duplication size & $4.96 \mathrm{Mb}$ & $0.901 \mathrm{Mb}$ & $0.3 \mathrm{Mb}$ & $\begin{array}{c}\text { Less than } 5 \% \text { have cytogenetically visible duplications. The rest } \\
\text { have microduplications ranging from } 0.3 \text { to } 4 \mathrm{Mb} \text {. }\end{array}$ \\
\hline Hypotonia & + & + & + & $50 \%-94 \%[4,5,9]$ \\
\hline $\begin{array}{l}\text { Developmental delay / intellectual } \\
\text { disability }\end{array}$ & + & + & + & $100 \%[4,5,9]$ \\
\hline Age at walking & - & - & 5 years old & 8 months -8 years $[4,9]$ \\
\hline Absent speech development & + & + & + & $79 \%-88 \%[4,5]$ \\
\hline Epilepsy & + & + & + (Lennox Gastaut Syndrome) & $52-54 \%[4,5]$ \\
\hline Age at onset of epilepsy & 18 months & 9 years old & 9 years old & Variable \\
\hline Progressive spasticity & - & + & + & $59-64 \%[4,5]$ \\
\hline Recurrent infections & + & + & + & $72-74 \%[4,5]$ \\
\hline Chronic constipation & + & - & + & $33-76 \%[4,5]$ \\
\hline Origin of duplication & $\begin{array}{c}\text { Maternally } \\
\text { inherited }\end{array}$ & $\begin{array}{l}\text { Maternally } \\
\text { inherited }\end{array}$ & Parental samples not available & N.A. \\
\hline
\end{tabular}

Table 1: Summary of clinical features of our patients and comparison with reported cases. 


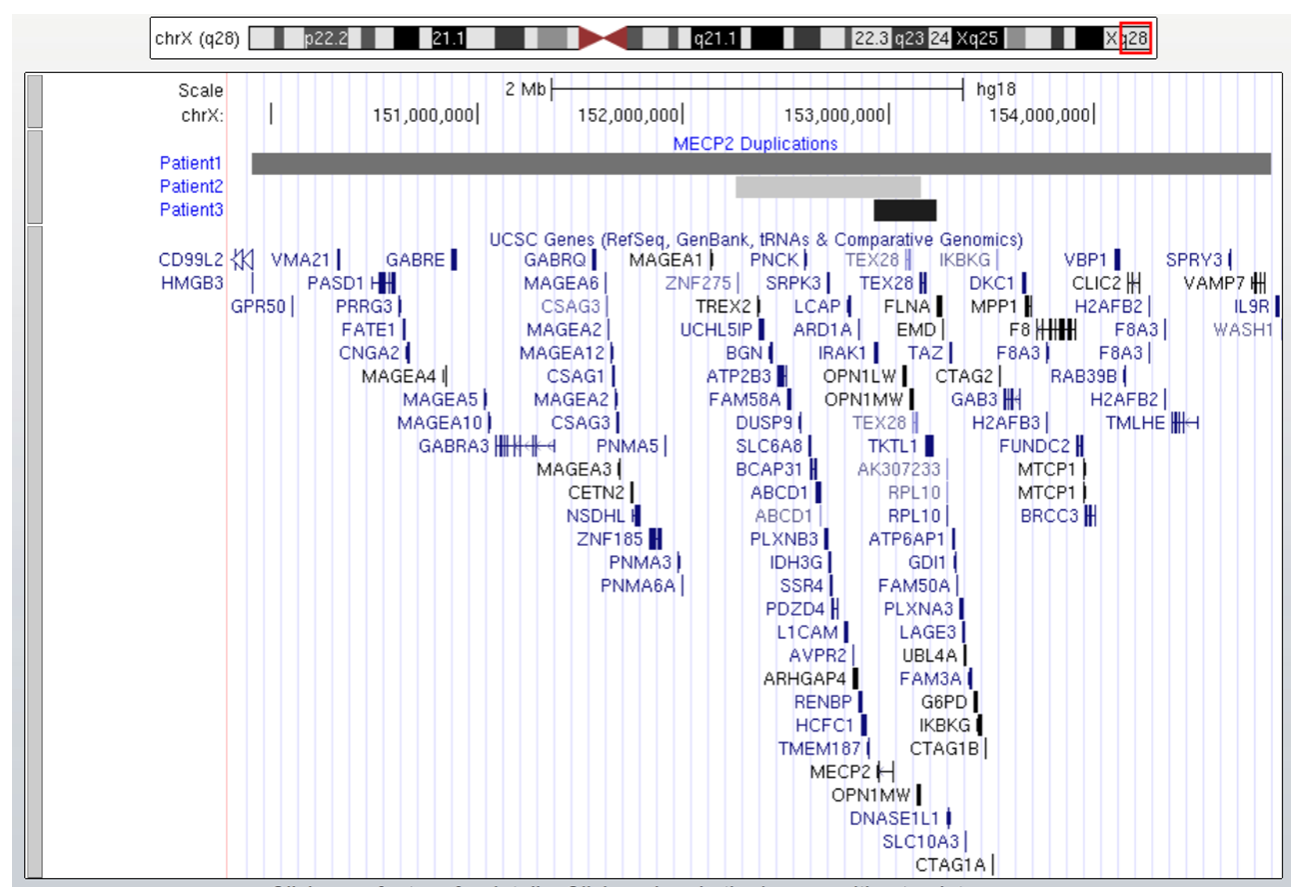

Figure 1: Screenshot of the UCSC Genome Browser showing the size of the duplications for the three patients and the genes affected. The minimum duplicated regions were ChrX:149,904,265 - 154,861,548 for Patient 1, ChrX:152,257,222 - 153158798 for Patient 2, and ChrX:152,930,233 - 153,230,284 for Patient 3 .

was frequently interrupted by prolonged hospitalizations for aspiration pneumonia and septicemia. He subsequently became bed-bound and required a gastrostomy for enteral feeding. He died at 16 years old while residing in institutionalized care.

\section{Molecular genetic analysis results}

CMA detected copy number gains at Xq28: 4.96 Mb for Patient 1, $1.90 \mathrm{Mb}$ for Patient 2, and $0.30 \mathrm{Mb}$ for Patient 3 (Figure 1).

Real-time PCR analysis targeting MECP2 confirmed the gains for Patients 1 and 2. Testing of parents showed that in Patient 1 and Patient 2 , the gains were inherited from their mothers while the fathers have normal copy number (Figure 2). Parental samples were not available for Patient 3.

\section{Discussion}

There are now more than 150 documented MECP2 duplication cases, with reported sizes ranging from $0.08 \mathrm{Mb}$ to $23 \mathrm{Mb}$ and breakpoints that are mostly unique [4,7]. This is in contrast to other recurrent microduplication syndromes which have recurrent breakpoints that result from homologous recombination in regions with low copy repeats. And for two of the three patients, we have further determined that the gains were inherited from mothers who were asymptomatic, likely because the regions with the gains were part of the X chromosomes which were inactivated and functionally inactive in the mothers.

The MECP2 gene encodes an essential epigenetic regulator in postnatal brain development. Mutations in the gene can cause Rett syndrome which is a postnatal neurodevelopmental disorder seen almost exclusively in females. Typical features include neuroregression following early normal growth and development, stereotypical hand movements, and problems with walking as well as social and language skills.
The neurological features of MECP2 duplication, which are present in our patients, include early hypotonia, developmental delay, epilepsy and limited or no speech. Due to axial hypotonia, gross motor milestones are delayed and patients may not start walking till 4 years of age [4]. Up to $80 \%$ of affected individuals do not develop speech, as seen in all three of our patients. Progressive spasticity, which leads to eventual poor mobility, was seen in Patient 2. Developmental regression which usually correlates with seizure onset is evident in Patient 3, possibly also contributed by his post-surgical complications. Abnormal movements mainly involving the upper limbs, observed in Patients 2 and 3, are also typical [5]. Seizures in children with MECP2 duplication syndrome often occur between the ages of 4 to 13 years, but isolated febrile seizures have been reported in younger children [8]. This is consistent with the clinical presentation of seizures in our cases. Seizure semiology is highly variable - generalized tonic-clonic, atonic, absence and myoclonic seizures have all been previously reported $[4,5,8,9]$.

Patient 3 was diagnosed with Lennox-Gastaut syndrome (LGS) based on his clinical presentation of refractory epilepsy and EEG findings. While refractory and drug-resistant epilepsy is often reported in children with MECP2 duplication syndrome, the majority of children are not diagnosed with a specific epilepsy syndrome $[8,10]$. The association of Lennox-Gastaut syndrome with MECP2 duplication has been reported in an adult study but not in paediatric reports [11].

Reported neuroimaging abnormalities include generalized ventricular dilatation, and hypoplasia of the corpus callosum and cerebellar vermis which are seen in Patient 1. A Dandy Walker variant (seen in Patient 1) has only been reported in one other patient [12].

About $75 \%$ of reported patients have recurrent respiratory infections, which are often the cause of a limited lifespan. All our patients had frequent hospitalizations for lower respiratory tract infections, with some episodes requiring invasive ventilation and intensive care admission. In a clinical study, $\mathrm{T}$ helper cells from children and mice 
(A)

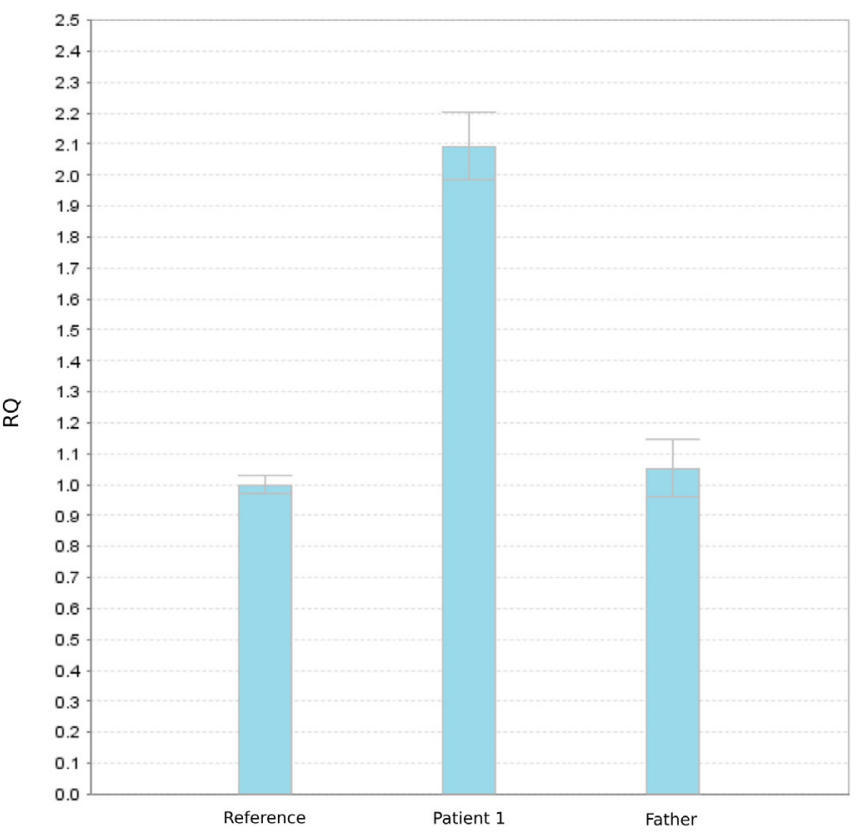

(c)

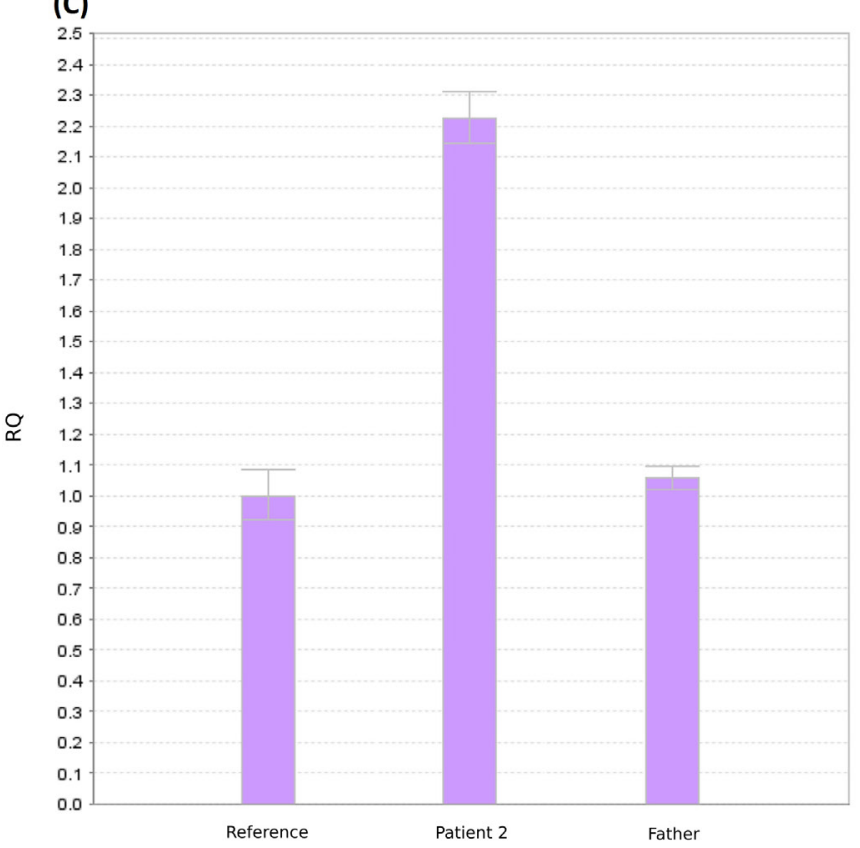

(B)

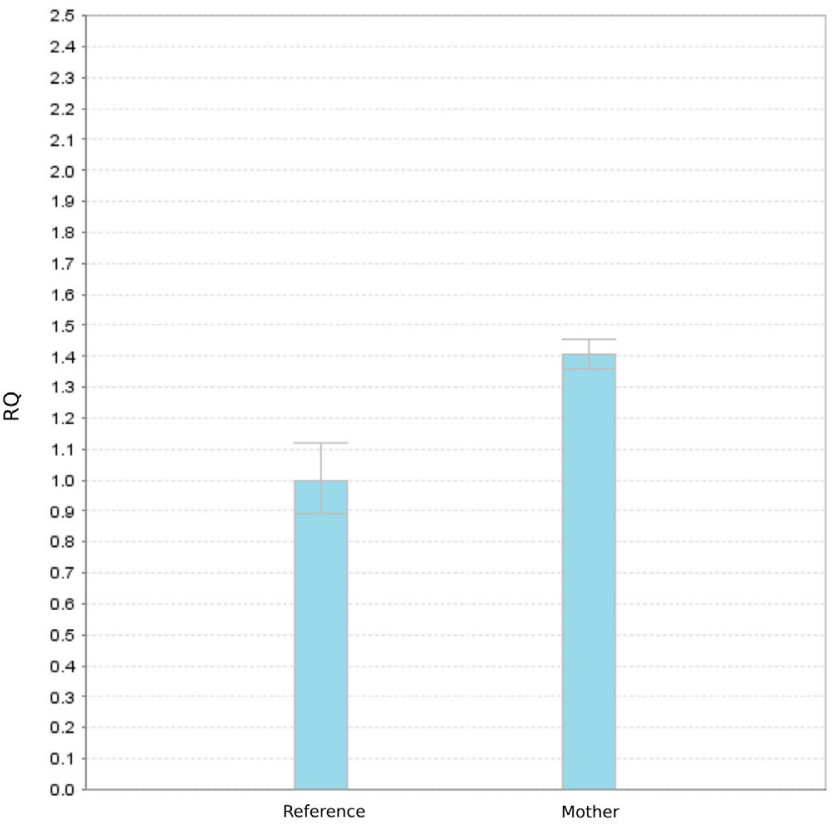

(D)

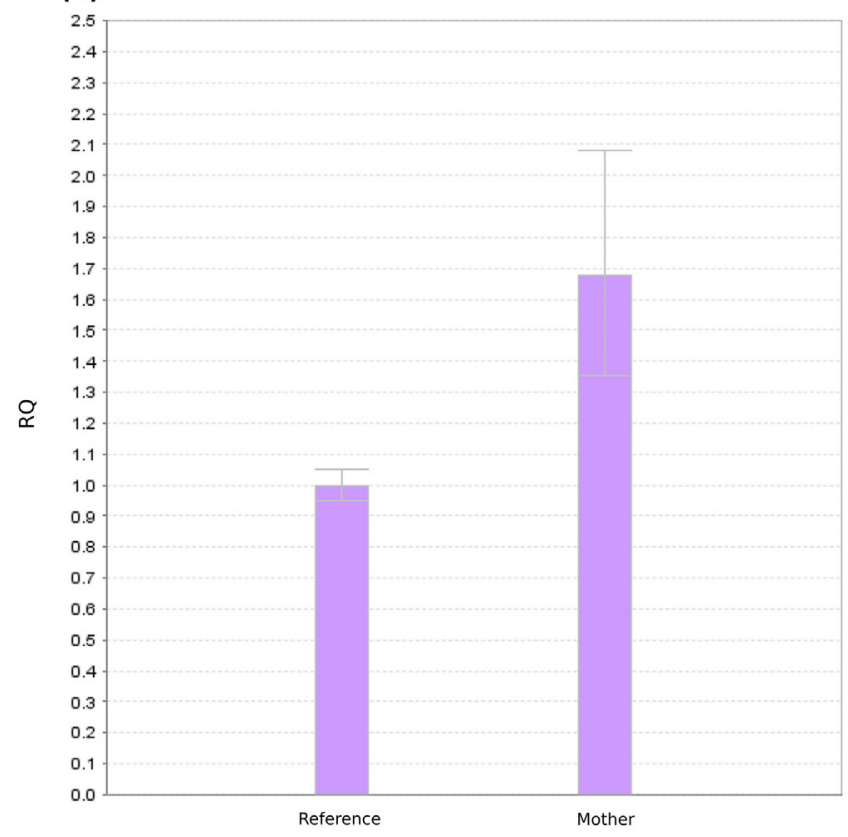

Figure 2: Confirmation of the Xq28 copy number gain by relative quantitation (RQ) of copy number of MECP2 using qRT-PCR for Patients 1 \& 2 . (A) Patient 1 and father compared to a male control. (B) Patient 1's mother compared to a female control. (C) Patient 2 and father compared to a male control. (D) Patient 2's mother compared to a female control.

with MECP2 duplication displayed impaired interferon-gamma secretion and $\mathrm{T}$ helper cell type 1 responses, suggesting a partially immunodeficient state [13]. This could explain the predisposition to recurrent respiratory infections. Of particular interest is the IRAK1 gene which is duplicated in almost all patients with MECP2 duplication syndrome, including all three of our patients. IRAK1 encodes an interleukin receptor-associated kinase that plays an important role in immunity against certain pyogenic bacteria by inducing inflammatory target gene expression [4]. However, immunological studies have not shown a clear role of IRAK1 duplication in the immunodeficiency observed in MECP2 duplication syndrome patients.

Patient 1 is of particular interest as the region of gain is large, including MECP2, FLNA, L1CAM, GDI1 and EMD. Increased FLNA gene dosage is thought to compromise enteric neuron development and affect neuronal migration, leading to the gastrointestinal symptoms of 
Citation: Chow C, Lai AHM, Brett MS, Ling S, Ha JS, et al. (2015) Cytogenomic Delineation and Clinical Characterization of Three Cases of MECP2 Duplication Syndrome. J Clin Med Genom 3: 125. doi: 10.4172/2472-128X.1000125

Page 5 of 5

chronic constipation or intestinal pseudo-obstruction [14]. The role of FLNA in chronic constipation is corroborated in our three patients: FLNA was duplicated in Patients 1 and 3 who had chronic constipation, while Patient 2 did not have FLNA duplication nor chronic constipation. For $L 1 C A M$ which is expressed predominantly in the nervous system, mutations have been associated with structural brain abnormalities such as hydrocephalus due to stenosis of the aqueduct of Sylvius and corpus callosum hypoplasia [15]. It is possible that Patient 1's MRI brain findings are related to involvement of this gene, as Patient 3's MRI was reported as normal (Patient 2's parents did not consent to MRI). GDI1 has been linked to X-linked intellectual disability,[16] while mutations in the Emerin $(E M D)$ gene lead to X-linked Emery-Dreifuss muscular dystrophy [17]. As gain-of-function mutations of EMD are yet to be reported, it is difficult to predict the influence that this gene duplication will have on our patient.

\section{Conclusion}

While our patients contribute to an increasing pool of $M E C P 2$ duplication syndrome individuals reported, it is likely that the frequency of this relatively new syndrome is under-reported. CMA can reliably diagnose this duplication syndrome as well as other microdeletion and microduplication syndromes, and is recommended as a first-tier genetic test in patients with unexplained intellectual disability. Making a specific genetic diagnosis supported by molecular data will allow physicians to provide appropriate anticipatory management, and more accurate information about prognosis and recurrence risks.

\section{Acknowledgements}

This work was funded by PPG/KKH12010-Theme3 and CG/RC001 from the National Medical Research Council, Ministry of Health, Republic of Singapore SHF/CS001/2008 from SingHealth Foundation. We also thank Dr Charles Lee for the use of his laboratory facilities at Brigham and Women's Hospital for the work on Patient 3.

\section{References}

1. Amir RE, Van den Veyver IB, Wan M, Tran CQ, Francke U, et al. (1999) Rett syndrome is caused by mutations in X-linked MECP2, encoding methyl-CpGbinding protein 2. Nat Genet 23: 185-188.

2. Meins M, Lehmann J, Gerresheim F, Herchenbach J, Hagedorn M, et al (2005) Submicroscopic duplication in Xq28 causes increased expression of the MECP2 gene in a boy with severe mental retardation and features of Rett syndrome. J Med Genet 42: e12.

3. Na ES, Nelson ED, Kavalali ET, Monteggia LM (2013) The impact of MeCP2 loss- or gain-of-function on synaptic plasticity. Neuropsychopharmacology 38 212-219.

4. Van Esch H (2012) MECP2 Duplication Syndrome. Mol Syndromol 2: 128-136

5. Ramocki MB, Tavyev YJ, Peters SU (2010) The MECP2 duplication syndrome. Am J Med Genet A 152A: 1079-1088.

6. Collins AL, Levenson JM, Vilaythong AP, Richman R, Armstrong DL, et al (2004) Mild overexpression of MeCP2 causes a progressive neurological disorder in mice. Hum Mol Genet 13: 2679-2689.

7. Sanmann JN, Bishay DL, Starr LJ, Bell CA, Pickering DL, et al. (2012) Characterization of six novel patients with MECP2 duplications due to unbalanced rearrangements of the X chromosome. Am J Med Genet A 158A: 1285-1291.

8. Vignoli A, Borgatti R, Peron A, Zucca C, Ballarati L, et al. (2012) Electroclinical pattern in MECP2 duplication syndrome: eight new reported cases and review of literature. Epilepsia 53: 1146-1155.

9. Echenne B, Roubertie A, Lugtenberg D, Kleefstra T, Hamel BC, et al. (2009) Neurologic aspects of MECP2 gene duplication in male patients. Pediatr Neuro 41: 187-191.

10. Caumes R, Boespflug-Tanguy O, Villeneuve N, Lambert L, Delanoe C, et al (2014) Late onset epileptic spasms is frequent in MECP2 gene duplication electroclinical features and long-term follow-up of 8 epilepsy patients. Eur $\mathrm{J}$ Paediatr Neurol 18: 475-481.

11. Lund C, Brodtkorb E, Røsby O, Rødningen OK, Selmer KK (2013) Copy number variants in adult patients with Lennox-Gastaut syndrome features. Epilepsy Res 105: 110-117.

12. Friez MJ, Jones JR, Clarkson K, Lubs H, Abuelo D, et al. (2006) Recurrent infections, hypotonia, and mental retardation caused by duplication of MECP2 and adjacent region in Xq28. Pediatrics 118: e1687-1695

13. Yang T, Ramocki MB, Neul JL, Lu W, Roberts L, et al. (2012) Overexpression of methyl-CpG binding protein 2 impairs $T(H) 1$ responses. Sci Transl Med 4: 163 ra158.

14. Clayton-Smith J, Walters S, Hobson E, Burkitt-Wright E, Smith R, et al. (2009) Xq28 duplication presenting with intestinal and bladder dysfunction and a distinctive facial appearance. Eur J Hum Genet 17: 434-443.

15. Weller S, Gärtner J (2001) Genetic and clinical aspects of X-linked hydrocephalus (L1 disease): Mutations in the L1CAM gene. Hum Mutat 18: $1-12$.

16. Castellví-Bel S, Milà M (2001) Genes responsible for nonspecific menta retardation. Mol Genet Metab 72: 104-108.

17. Brown CA, Scharner J, Felice K, Meriggioli MN, Tarnopolsky M, et al. (2011) Novel and recurrent EMD mutations in patients with Emery-Dreifuss muscular dystrophy, identify exon 2 as a mutation hot spot. J Hum Genet 56: 589-594. 